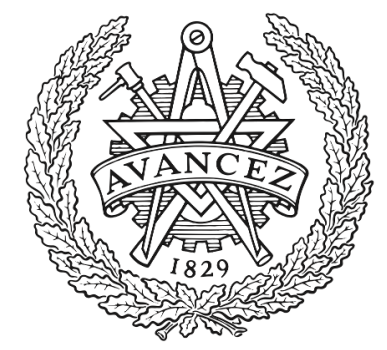

CHALMERS

UNIVERSITY OF TECHNOLOGY

\title{
Isotope identity experiments in JET-ILW with $H$ and D L-mode plasmas
}

Downloaded from: https://research.chalmers.se, 2020-06-30 12:01 UTC

Citation for the original published paper (version of record):

Maggi, C., Weisen, H., Casson, F. et al (2019)

Isotope identity experiments in JET-ILW with $\mathrm{H}$ and D L-mode plasmas

Nuclear Fusion, 59(7)

http://dx.doi.org/10.1088/1741-4326/ab1ccd

N.B. When citing this work, cite the original published paper. 


\title{
Isotope identity experiments in JET-ILW with $\mathrm{H}$ and $\mathrm{D}$ L-mode plasmas
}

\author{
C.F. Maggi ${ }^{1, a}{ }^{\oplus}$, H. Weisen ${ }^{2}$, F.J. Casson ${ }^{1}$, F. Auriemma ${ }^{3}$, R. Lorenzini ${ }^{3}$, \\ H. Nordman ${ }^{4}$, E. Delabie ${ }^{5}$, F. Eriksson ${ }^{4}$, J. Flanagan ${ }^{1}$, D. Keeling ${ }^{1}$, \\ D. King ${ }^{1}$, L. Horvath ${ }^{6}$, S. Menmuir ${ }^{1}$, A. Salmi ${ }^{7}$, G. Sips ${ }^{8}$, T. Tala ${ }^{7}$, \\ I. Voitsekhovich ${ }^{1}$ and JET Contributors ${ }^{b}$
}

EUROfusion Consortium, JET, Culham Science Centre, Abingdon, OX14 3DB, United Kingdom of Great Britain and Northern Ireland

${ }^{1}$ CCFE, Culham Science Centre, Abingdon OX14 3DB, United Kingdom of Great Britain and Northern Ireland

${ }^{2}$ SPC, Ecole Polytechnique Federale de Lausanne, Switzerland

3 Consorzio RFX, Corso Stati Uniti 4, I-35127 Padova, Italy

${ }^{4}$ Chalmers University of Technology, Göteborg, Sweden

5 Oak Ridge National Laboratory, Oak Ridge, Tennessee, United States of America

${ }^{6}$ Department of Physics, York Plasma Institute, University of York, York YO10 5DD,

United Kingdom of Great Britain and Northern Ireland

7 VTT, FI-02044 VTT, Espoo, Finland

${ }^{8}$ European Commission, Brussels, Belgium

E-mail: Costanza.Maggi@ukaea.uk

Received 17 January 2019, revised 26 March 2019

Accepted for publication 26 April 2019

Published 7 June 2019

\begin{abstract}
NBI-heated L-mode plasmas have been obtained in JET with the Be/W ITER-like wall (JETILW) in $\mathrm{H}$ and $\mathrm{D}$, with matched profiles of the dimensionless plasma parameters, $\rho^{*}, \nu^{*}, \beta$ and $\mathrm{q}$ in the plasma core confinement region and same $T_{\mathrm{i}} / T_{\mathrm{e}}$ and $Z_{\text {eff. }}$. The achieved isotope identity indicates that the confinement scale invariance principle is satisfied in the core confinement region of these plasmas, where the dominant instabilities are Ion Temperature Gradient (ITG) modes. The dimensionless thermal energy confinement time, $\Omega_{\mathrm{i}} \tau_{\mathrm{E}, \mathrm{th}}$, and the scaled core plasma heat diffusivity, A $\chi_{\mathrm{eff}} / B_{\mathrm{T}}$, are identical in $\mathrm{H}$ and $\mathrm{D}$ within error bars, indicating lack of isotope mass dependence of the dimensionless L-mode thermal energy confinement time in JET-ILW. Predictive flux driven simulations with JETTO-TGLF of the H and D identity pair is in very good agreement with experiment for both isotopes: the stiff core heat transport, typical of JET-ILW NBI heated L-modes, overcomes the local gyro-Bohm scaling of gradient-driven TGLF, explaining the lack of isotope mass dependence in the confinement region of these plasmas. The effect of $E \times B$ shearing on the predicted heat and particle transport channels is found to be negligible for these low beta and low momentum input plasmas.
\end{abstract}

Keywords: tokamak plasmas, isotope identity, JET-ILW

(Some figures may appear in colour only in the online journal)

\section{Introduction}

Dimensionless identity experiments test the invariance of plasma physics to changes in the dimensional plasma parameters,

${ }^{a}$ Author to whom any correspondence should be addressed.

${ }^{\mathrm{b}}$ See the author list of Litaudon et al [25]. e.g. density and temperature, when the canonical dimensionless plasma physics parameters $\rho^{*}, \nu^{*}, \beta, q, \ldots$ are conserved [1, 2]. Plasmas with dissimilar dimensional parameters but identical dimensionless parameters should have identical transport, with the appropriate normalization to make it dimensionless [2]. However, conditions at the plasma boundary, such as influx 
of neutral particles, may introduce additional physics, potentially invalidating this approach. It is therefore important to test experimentally the validity of this principle, which is at the heart of extrapolations of plasma transport properties to future devices. The inverse aspect ratio $\varepsilon=a / R$ and $\kappa$, the ratio of plasma height and width, also need to be constant. Following the literature, e.g. [2], the dimensionless thermal energy confinement time has the general form:

$$
\Omega_{\mathrm{i}} \tau_{\mathrm{E}, \mathrm{th}}=F\left(\rho^{*}, \nu^{*}, \beta, \ldots\right)
$$

with $\Omega_{\mathrm{i}} \sim e B / A$ the ion cyclotron frequency ( $e$ is the particle charge). Typically, the function $\mathrm{F}$ is expressed in terms of the product of power laws of the plasma dimensionless parameters [2]:

$$
\Omega_{\mathrm{i}} \tau_{\mathrm{E}, \mathrm{th}} \sim\left(\rho^{*-\alpha_{\rho}} \beta^{-\alpha_{\beta}} \nu^{*-\alpha_{\nu}} q^{-\alpha_{q}} A^{-\alpha_{\mathrm{A}}}\right) .
$$

$\rho^{*} \sim \sqrt{ }\left(A T_{\mathrm{i}}\right) /\left(a B_{\mathrm{T}}\right)$ is the Larmor radius of thermal ions normalized to the plasma minor radius (with $A=m_{\mathrm{i}} / m_{\mathrm{p}}$ the isotope ion mass normalized to the proton mass, $T_{\mathrm{i}}$ the ion temperature, $a$ the plasma minor radius and $B_{\mathrm{T}}$ the toroidal magnetic field on axis); $\beta \sim n T / B^{2}$ is the plasma pressure normalized to the magnetic pressure (with $n$ the plasma density); $\nu^{*} \sim n_{\mathrm{e}} R Z_{\mathrm{eff}} / T_{\mathrm{e}}^{2}$ is the electron-ion collision frequency normalized to the thermal ion bounce frequency (with $Z_{\text {eff }}$ the plasma effective charge, $R$ the major radius); $q \sim B /(I R)$ is the safety factor (with $I$ the plasma current).

The favourable dependence of energy and particle transport on the main ion isotope mass is of fundamental interest for the understanding of turbulent transport and, therefore, for accurate predictions of confinement from present day tokamak experiments to future burning devices. While future experiments, such as ITER, will operate in Deuterium-Tritium mixtures, today's tokamak experiments typically use a single hydrogen isotope (predominantly deuterium). Complete theoretical understanding of the favourable isotopic dependence of transport and confinement still remains elusive, but recently new impetus was injected in this field of research by a series of isotope experiments in JET with the ITER-like Be/W wall (JET-ILW) in preparation for a second D-T experiment. While most studies investigate the isotope scaling of confinement in dimensional experiments, we follow here the Connor-Taylor scale invariance approach [1]. Isotope identity experiments exploit the change in isotope ion mass to obtain plasmas with identical dimensionless profiles in the same tokamak. In order to keep $\rho^{*}, \beta, \nu^{*}$ and $q$ profiles fixed when also varying the isotope mass, the plasma current, toroidal magnetic field and the density and temperature must scale, respectively, as: $I_{\mathrm{P}}, B_{\mathrm{T}}$ $\sim A^{3 / 4} ; n \sim A$ and $T \sim \sqrt{ } A[3,4]$. Accordingly, for equal scaled energy confinement times $B \tau_{\mathrm{E}, \mathrm{th}} / A$, the absorbed input power must scale as $P_{\mathrm{abs}}=W_{\mathrm{th}} / \tau_{\mathrm{E} \text {,th }} \sim B_{\mathrm{T}}^{5 / 3}$ (or $\sim A^{5 / 4}$ ), where $W_{\text {th }}$ is the thermal stored energy, and the heat diffusivity must scale as $\chi \sim B / A$, with $\chi$ defined in terms of the temperature gradient and the heat flux $q=-n \chi \nabla T$.

An isotope identity experiment was first carried out in the JET tokamak with C wall (JET-C). Type I ELMy H-modes were obtained in $\mathrm{H}$ (1MA/1T, H-NBI) and D (1.7MA/1.7T, $\mathrm{D}-\mathrm{NBI}$ ), with average plasma triangularity $\delta=0.3$, with matching $\rho^{*}, \nu^{*}, \beta$ and q profiles [3]. Remarkably, it was found that the scaled thermal energy confinement time, $B \tau_{\mathrm{E}, \mathrm{th}} / A$, the scaled ELM frequency, $A f_{\mathrm{ELM}} / B$, and the scaled sawtooth frequency, $A f_{\text {saw }} / B$, were all matched within error bars in the $\mathrm{H}$ and D plasmas, indicating that the invariance principle was satisfied throughout the entire plasma radius, despite the different physical processes in the plasma centre, core confinement and edge regions [3]. We note that the match in scaled ELM frequency and scaled sawtooth frequency in $\mathrm{H}$ and D is not in the original theory of [1] and could have been fortuitous in the experiments reported in [3]. We also note that in the JET-C experiments the plasma isotope purity in $\mathrm{H}$, $n_{\mathrm{H}} /\left(n_{\mathrm{H}}+n_{\mathrm{D}}\right)=0.89$, was not as good as can be obtained in JET-ILW (see section 3), the Be/W metallic wall having demonstrated significant reduction in main fuel retention [4] and faster isotope wall change-over.

When the isotope identity has been achieved, e.g. in the $\mathrm{H}$ and D discharge pair of [3], one can extract the value of the exponent $\alpha_{\mathrm{A}}$ yielding the isotope mass scaling of the dimensionless thermal energy confinement time $\Omega_{\mathrm{i}} \tau_{\mathrm{E}, \mathrm{th}} \sim A^{-\alpha \mathrm{A}}$, since the quantity $\left(\rho^{*-\alpha \rho} \beta^{-\alpha \beta} \nu^{*-\alpha \nu} q^{-\alpha q} \ldots\right.$ ) in equation (2) is kept constant. The JET-C isotope identity experiment in $\mathrm{H}$ and $\mathrm{D}$ in type I ELMy H-mode yielded a weak, positive isotope mass scaling $\Omega_{\mathrm{i}} \tau_{\mathrm{E}, \mathrm{th}} \sim A^{0.14}$ [2]. This was found to be largely consistent with the lack of mass dependence of (dimensional) global energy confinement time, $\tau_{\mathrm{E}, \mathrm{th}} \sim A^{0.03 \pm 0.10}$, obtained in JET-C type I ELMy H-modes in H, D, T and D-T at similar density and input power. The latter resulted from the combination of strong, positive mass dependence for $W_{\text {PED }}$ (pedestal stored energy) and weak, negative mass dependence in the plasma core, $\tau_{\text {th,core }} \sim A^{-0.16}$ [5]. The scaled local heat diffusivities from power balance, $A \chi_{\mathrm{eff}} / B$, were also found to be similar in $\mathrm{H}$ and $\mathrm{D}$, within experimental uncertainties in the TRANSP power balance, with $\chi_{\text {eff }}$ the one-fluid thermal diffusivity, since a species resolved power balance analysis was not possible in [3].

The isotope identity technique was revisited in recent experiments with $\mathrm{H}$ and D plasmas in JET-ILW. Additionally, compared to the earlier JET-C experiments, improved core and edge electron profile diagnosis with HRTS is available [6]. This paper reports on the experiments in the L-mode regime: the experimental results and interpretative TRANSP analysis are described in section 2, predictive core plasma transport modelling is presented in section 3 , followed by conclusions and outlook in section 4.

In ELMy H-modes, isotope identity experiments must also demonstrate the profile match in the pedestal region, which strongly influences the global energy confinement for stiff core heat transport. Moreover, the evidence from JET-ILW is that the strong, favourable isotope mass dependence of $\tau_{\mathrm{E} \text {,th }}$ in type I ELMy H-modes originates at the pedestal [7]. As mentioned earlier, conditions at the plasma boundary, such as influx of neutral particles across the LCFS and into the edge transport barrier, may introduce additional physics, so that the canonical plasma physics parameters may no longer be sufficient to describe the plasma transport properties. This could potentially invalidate the approach of the confinement scale 

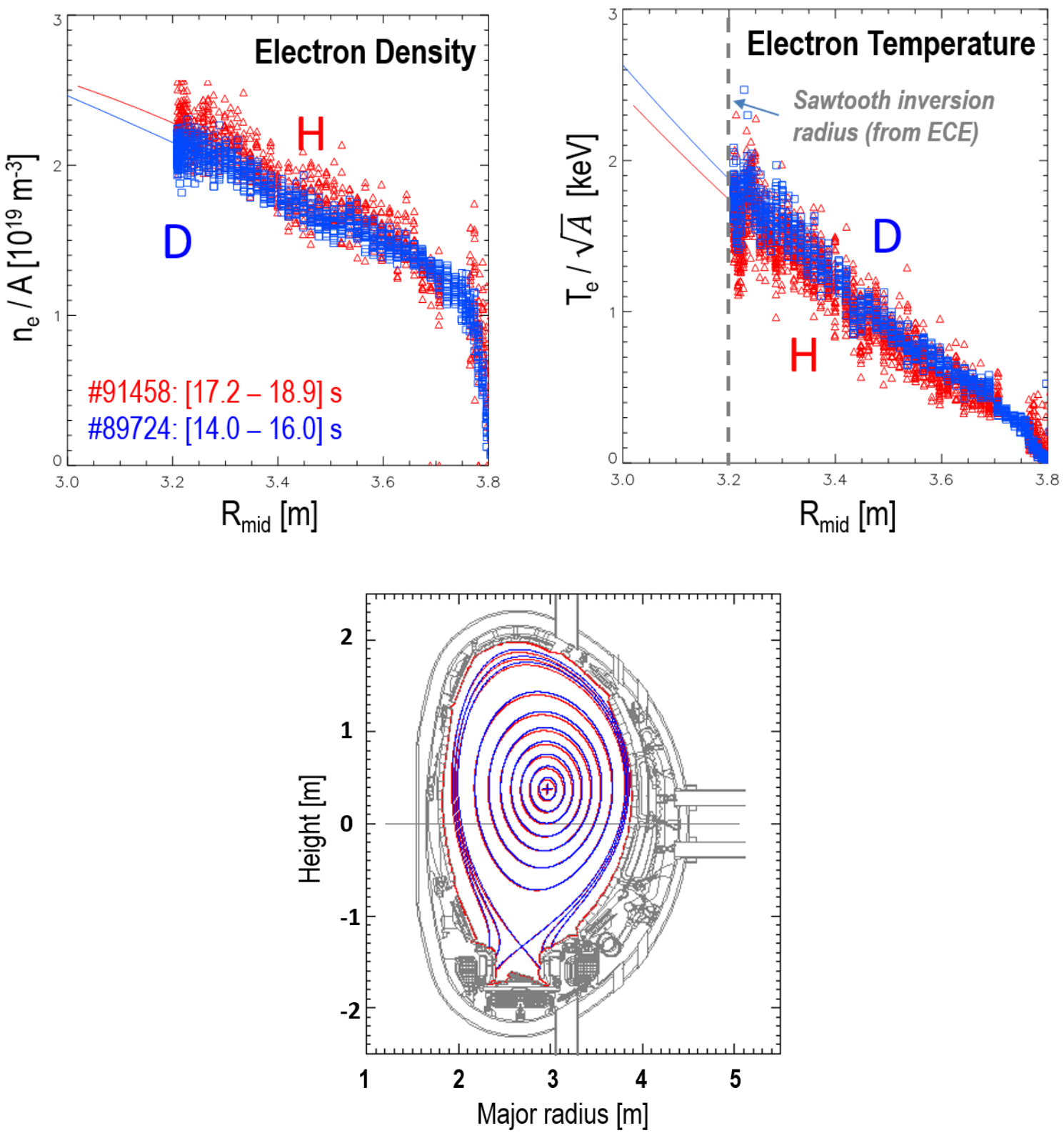

Figure 1. Scaled $n_{\mathrm{e}}(a)$ and $T_{\mathrm{e}}(b)$ profiles from HRTS for the JET-ILW L-mode isotope identity pair in H (\#91458, red) and D (\#89724, blue) versus $R_{\text {mid }}$, the major radius at $z$ of magnetic axis. The data are composite profiles in the steady time interval analysed (noted in figure $(a)$ ) and the solid lines correspond to the fits to the data. $T_{\mathrm{i}}=T_{\mathrm{e}}$ within experimental uncertainties of the $T_{\mathrm{i}}$ measurements from CXRS $( \pm 10 \%)$. (c) Matched plasma equilibria in $\mathrm{H}$ and $\mathrm{D}$, showing the divertor configuration with both strike points on the vertical targets.

invariance principle. To explore these issues, isotope identity experiments were also performed in H and D type I ELMy H-modes in JET-ILW. However, further optimization of the profiles matches is sought in the upcoming JET campaign and these studies will be reported in a future work.

\section{JET-ILW L-mode isotope identity experiment and interpretative analysis}

An L-mode isotope identity pair was achieved in JET-ILW at $I_{\mathrm{P}} / B_{\mathrm{T}}=2.46 \mathrm{MA} / 2.95 \mathrm{~T}$ in $\mathrm{D}$ and $1.44 \mathrm{MA} / 1.74 \mathrm{~T}$ in $\mathrm{H}$, $q_{95}=3.4$, low plasma triangularity $\delta=0.2$. The chosen divertor configuration was with both strike points on the divertor vertical targets, as shown in figure 1 , in order to maximize the $\mathrm{L}-\mathrm{H}$ power threshold at a given plasma density and toroidal magnetic field [8] and thus maximize the L-mode domain, in particular in view of comparison with Tritium discharges, which are foreseen in future JET-ILW experiments. The auxiliary heating was provided by neutral beam injection (NBI), with D-NBI (beam energy: 82-91 keV) and H-NBI (beam energy: 64-71 keV), respectively. The excellent isotope purity in these plasmas, $n_{\mathrm{H}} /\left(n_{\mathrm{H}}+n_{\mathrm{D}}\right)>98 \%$, is a feature of 

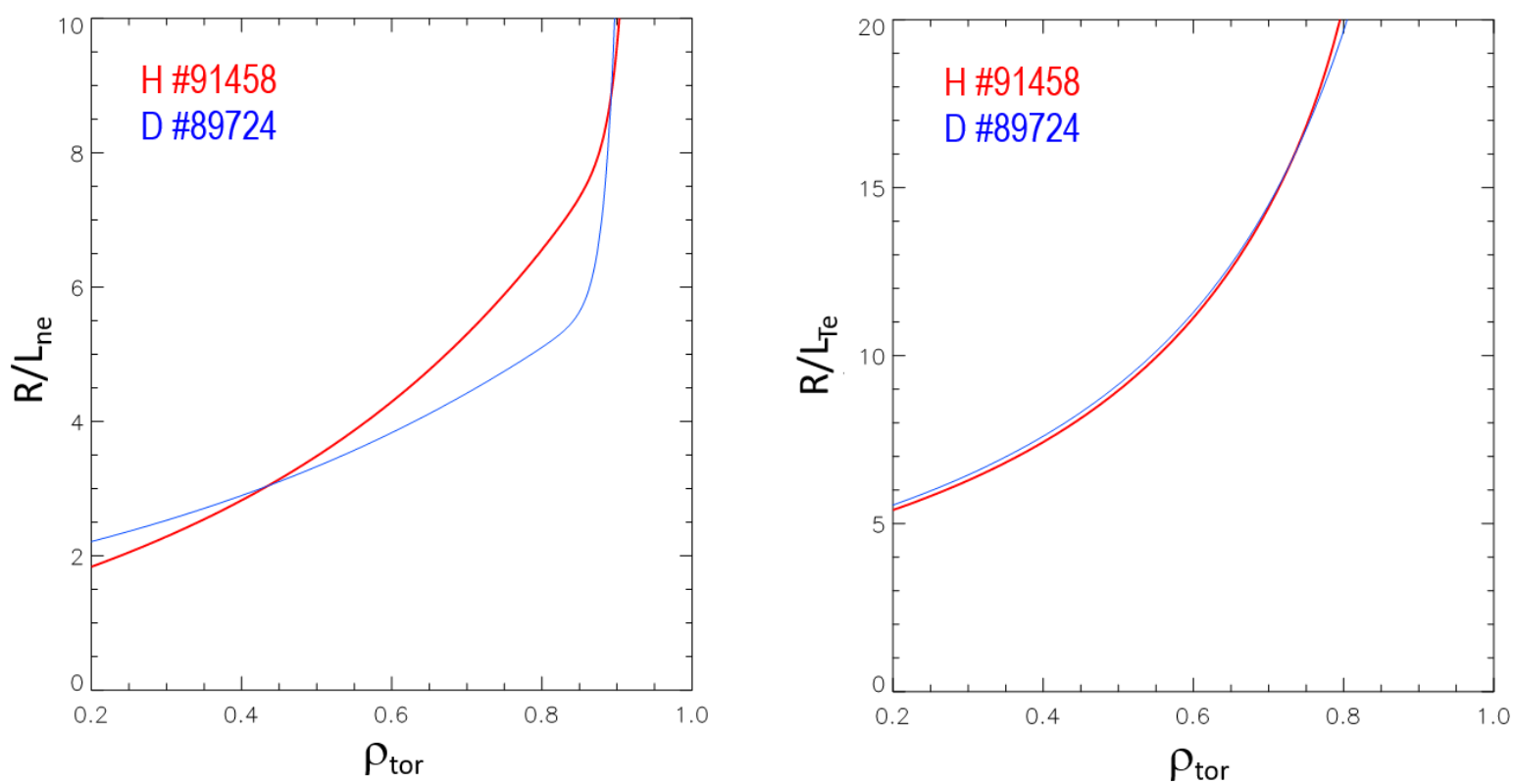

Figure 2. Normalized electron density (left) and temperature (right) gradient lengths of the JET-ILW L-mode isotope identity pair in $\mathrm{H}$ (red) and D (blue) of figure 1 versus $\rho_{\text {tor }}=\sqrt{ } \Phi_{\mathrm{N}}$, with $\Phi_{\mathrm{N}}$ the normalized toroidal flux. The estimated errors on temperature and density gradient lengths are $\Delta\left(R / L_{T_{\mathrm{e}}}\right) \sim 1.5$ and $\Delta\left(R / L_{n_{\mathrm{e}}}\right) \sim 2.5$, respectively, as estimated by Gaussian process regression analysis [11], thus the differences between the two shots are within this uncertainty.

the ITER-like Be/W wall and a result of the long (six-week) $\mathrm{H}$ experimental campaign. The $n_{\mathrm{H}} /\left(n_{\mathrm{H}}+n_{\mathrm{D}}\right)$ ratio was continuously monitored by edge (divertor and midplane) Balmer- $\alpha$ spectroscopy, sub-divertor neutral gas analysis, a core neutral particle analyser and total neutron rate (the latter was particularly useful as a measure of plasma core $n_{\mathrm{H}} /\left(n_{\mathrm{H}}+n_{\mathrm{D}}\right)$ fractions during mixed H/D plasma experiments). After the last introduction of $\mathrm{D}$ (by pellets) into plasma discharges, the $\mathrm{D}$ concentration dropped to $1 \%$ within three $\mathrm{H}$ plasma discharges and remained constant at this level for the full $\mathrm{H}$ campaign [9].

Figures $1(a)$ and $(b)$ show the good match in the scaled kinetic profiles $n_{\mathrm{e}} / A$ and $T_{\mathrm{e}} / \sqrt{ } A$, measured by HRTS, for the $\mathrm{H}$ and $\mathrm{D}$ discharge pair. The data are composite profiles in the steady time intervals chosen for the analysis, which correspond to $10 \times \tau_{\mathrm{E}, \mathrm{th}}$. For both $\mathrm{H}$ and $\mathrm{D}$ plasmas, $T_{\mathrm{i}}=T_{\mathrm{e}}$ within uncertainties of the $T_{\mathrm{i}}$ measurements $( \pm 10 \%)$ from CXRS [10], obtained from C VI, $n=8-7$ transition for the D pulse and from $\mathrm{Ne} X, n=11-10$ transition for the H pulse, which used trace Ne puffs to enable core CXRS measurements. The Ne concentration profile for pulse \#91458, as estimated by core CXRS, varies from $0.2 \%$ in the plasma core to $0.07 \%$ at the plasma edge. The normalized density and temperature gradient lengths, $R / L_{\mathrm{ne}}=-R\left(\mathrm{~d} n_{\mathrm{e}} / \mathrm{d} r\right) / n_{\mathrm{e}}$ and $R / L_{T_{\mathrm{e}}}=-R\left(\mathrm{~d} T_{\mathrm{e}} / \mathrm{d} r\right) / T_{\mathrm{e}}$, corresponding to the profiles of figure 1 are derived from the fits to the composite profile data and are compared in figures $2(a)$ and $(b)$, showing good agreement in $\mathrm{H}$ and $\mathrm{D}$, especially for $R / L_{T_{\mathrm{e}}}$. The estimated errors on temperature and density gradient lengths are $\Delta\left(R / L_{T_{\mathrm{e}}}\right) \sim 1.5$ and $\Delta\left(R / L_{n_{\mathrm{e}}}\right) \sim 2.5$, respectively, as estimated by Gaussian process regression analysis [11], thus the differences between the two shots are within this uncertainty. In particular, the difference in density gradient length between $\mathrm{H}$ and $\mathrm{D}$ is not likely to have a significant impact on turbulence drive, since the turbulence is dominated by ITG modes in the core of these plasmas, as explained in section 3.

The profiles of the matched dimensionless plasma parameters of the $\mathrm{H}$ and D L-mode pair are shown in figure 3. Following the literature [1-3], the normalized ion Larmor radius $\rho^{*}$, the normalized collisionality $\nu^{*}$ and the normalized thermal pressure $\beta$ profiles have been calculated using the following definitions:

$$
\begin{gathered}
\rho^{*}=4.57 \times 10^{-3}\left(A T_{\mathrm{i}}\right)^{\frac{1}{2}} /\left(a B_{\mathrm{T}}\right) \\
\nu^{*}=0.0011 n_{\mathrm{e}} R q_{95} Z_{\mathrm{eff}} /\left(\varepsilon^{3 / 2} T_{\mathrm{e}}^{2}\right) \\
\beta=\left(p_{\mathrm{e}}+p_{\mathrm{i}}\right) /\left(B_{\mathrm{T}}^{2} / 2 \mu_{0}\right)
\end{gathered}
$$

where $B_{\mathrm{T}}(\mathrm{T})$ is the toroidal magnetic field on axis, $T_{\mathrm{i}}$ and $T_{\mathrm{e}}$ are in $\mathrm{keV}, n_{\mathrm{e}}$ is in $10^{19} \mathrm{~m}^{-3}$ and $p_{\mathrm{e}}$ and $p_{\mathrm{i}}$ are the electron and ion thermal pressure profiles, respectively. The ion thermal pressure is calculated from $T_{\mathrm{i}}=T_{\mathrm{e}}$ and $n_{\mathrm{i}}=n_{\mathrm{e}}\left(5-Z_{\mathrm{eff}}\right) / 4$, assuming Beryllium as the main impurity. The $q$-profile is matched by keeping constant plasma shape and scaling $I_{\mathrm{P}}$ and $B_{\mathrm{T}}$ proportionally to $A^{3 / 4}$, as introduced in section 1 . We note that with this choice of dimensionless parameters the isotope ion mass only appears in the normalized ion Larmor radius $\rho^{*}$ and not in the collisionality, which is here the electronion collisionality. An alternative choice of $\nu^{*}$, using the ionion collisionality $\nu_{\mathrm{ii}} \sim 1 / \sqrt{ } A$, which depends on the ion mass, could also be investigated in future studies, by designing a new isotope identity experiment where this parameter is matched (together with $\rho^{*}, \beta, \mathrm{q}, \ldots$ ). The isotope mass also plays a role in the electron-ion thermal equipartition term, $P_{\mathrm{ei}} \sim 1 / \mathrm{A}$, which is important for transport. Although in the experiments described here, with $T_{\mathrm{i}}=T_{\mathrm{e}}$ and similar direct ion and electron heating provided by NBI, this term is of minor importance in 

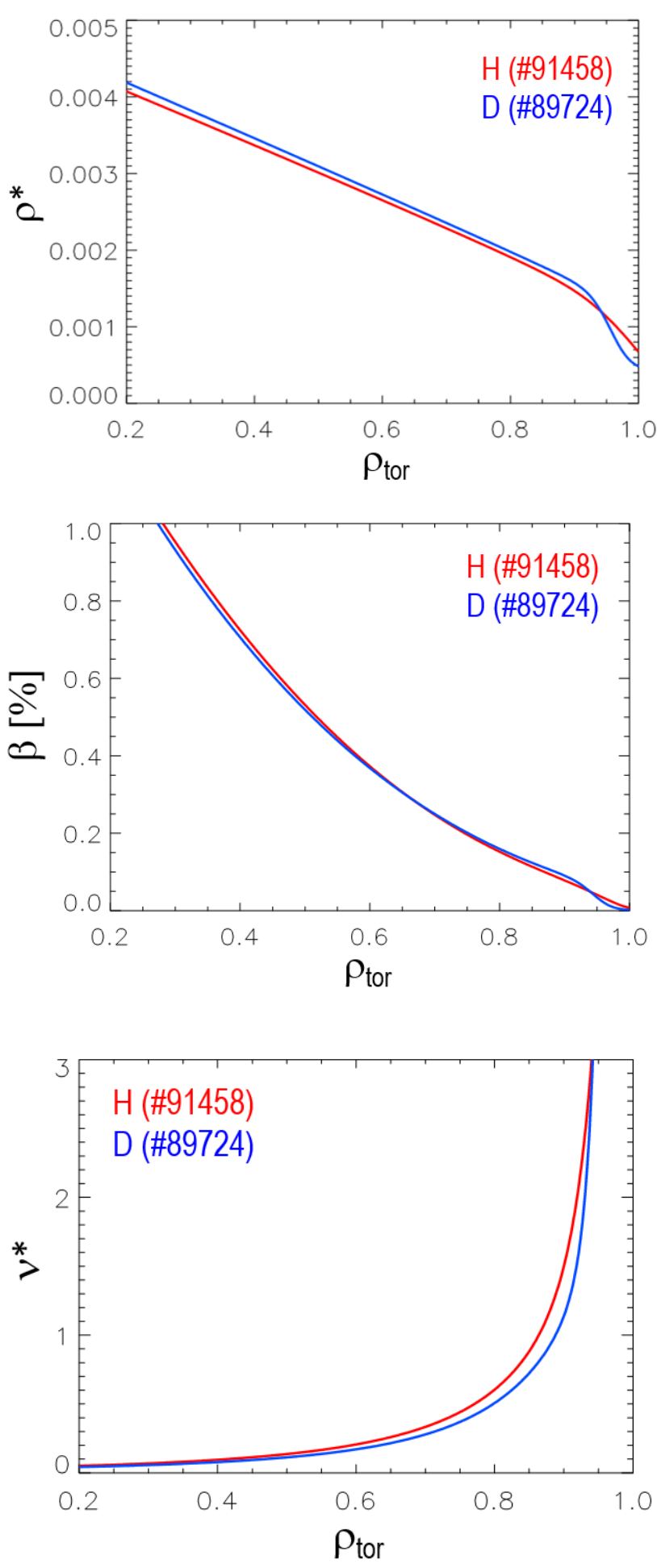

Figure 3. $\rho^{*}, \beta$, and $\nu^{*}$ profiles versus $\rho_{\text {tor }}$ for the JET-ILW L-mode isotope identity pair in $\mathrm{H}$ (red) and $\mathrm{D}$ (blue).

the overall power balance, it should be examined in isotope identity experiments with significant decoupling of $T_{\mathrm{e}}$ and $T_{\mathrm{i}}$.

The choice of NB energies, quoted above, with slightly lower energies in $\mathrm{H}$, was optimized before the experiment with scans in H-NBI and D-NBI voltages using the neutral beam deposition code PENCIL [12], to try and obtain the best match in particle source and beam heating profiles in $\mathrm{H}$ and
$\mathrm{D}$ (beam absorption changes when beam voltage and isotope mass change, in addition the NB full/half/third energy fractions are different for H-NBI and D-NBI). For the H and D isotope identity pair, the cumulative volume integrals of the total NB heating and particle sources calculated by TRANSP/ NUBEAM [13], and appropriately scaled by $B_{T}^{5 / 3}$ and by A respectively, are shown in figure 4. Similar cumulative NB heating profiles were achieved in $\mathrm{H}$ and $\mathrm{D}$, as shown in figure 4(a). Figure 4(b) shows that the scaled cumulative NB particle source profile is somewhat larger in $\mathrm{H}$. The line averaged $Z_{\text {eff }}$, measured by visible Bremsstrahlung (with Be the main impurity), is also similar in $\mathrm{H}$ and $\mathrm{D}$, as shown in table 1 . We thus conclude that the dimensionless identity is achieved in the L-mode core confinement region. The scaled thermal energy confinement times $B_{\mathrm{T}} \tau_{\mathrm{E}, \mathrm{th}} / A$ and core plasma effective heat diffusivities (from TRANSP power balance), $A \chi_{\mathrm{eff}} / B_{\mathrm{T}}$, were found to be similar in $\mathrm{H}$ and $\mathrm{D}$ within experimental uncertainties, thus satisfying the confinement scale invariance principle, as shown in table 1 and figure 5, respectively.

Finally, we note a mismatch (less than a factor of 2) in Mach number $M \sim \omega_{\text {tor }} R \sqrt{ } m_{\mathrm{i}} / \sqrt{ } k T_{\mathrm{e}}$ (toroidal rotation velocity normalized to ion sound speed, with $v_{\text {tor }}=R \omega_{\text {tor }}$ ) between the $\mathrm{H}$ and $\mathrm{D}$ shots. For reference, the Mach number values at $\rho_{\text {tor }}=0.5$ are $M=0.25$ in $\mathrm{H}$ and $M=0.35$ in $\mathrm{D}$, respectively. However, as will be shown in section 3 by predictive core transport modelling, the mismatch in $M$ profiles is not significant in this case and does not invalidate the achieved dimensionless profiles identity.

The sawtooth inversion radius, obtained from the ECE diagnostic, is located at $\rho_{\text {tor }} \sim 0.22-0.26$, where $q_{\psi} \sim 1$. The scaled sawtooth frequencies were different in the JET-ILW L-mode identity pair, with $A f_{\text {saw }} / B_{\mathrm{T}}=7.5 \mathrm{~Hz} \mathrm{~T}^{-1}$ in $\mathrm{H}$ and 4.7 $\mathrm{Hz} \mathrm{T}^{-1}$ in $\mathrm{D}$, almost twice in $\mathrm{H}$ than in $\mathrm{D}$. On the other hand, as noted previously in section 1 , in the JET-C type I ELMy H-mode isotope identity also the scaled sawtooth frequencies were matched in $\mathrm{H}$ and in $\mathrm{D}$ [3]. In dimensional L-mode experiments in JET-C, with NBI heating (6 MW) and limiter discharges (3.1 MA/2.9 T), $f_{\text {saw }}(\mathrm{H})$ was approximately twice than $f_{\text {saw }}(\mathrm{D})$ [14]. In the JET-ILW L-mode NBI power scans at constant density in $\mathrm{H}$ and $\mathrm{D}$, at 2.5MA/3.0T, reported in [7], the sawteeth were more frequent in $\mathrm{H}$ than in D at low $P_{\mathrm{NBI}}$, while $f_{\text {saw }}$ became similar in the two isotopes at the highest powers in the scan (7-9 MW). These different results warrant further investigation, which is however outside the scope of this paper.

The scaled energy confinement times of the $\mathrm{H}$ and $\mathrm{D}$ isotope identity pair yield $\Omega_{\mathrm{i}} \tau_{\mathrm{E} \text {,th }} \sim A^{0.05 \pm 0.1}$, indicating no isotope mass dependence for the dimensionless thermal energy confinement time. We may therefore ask if this result is consistent with the finding $\tau_{\mathrm{E}, \mathrm{th}} \sim A^{0.15 \pm 0.02}$ derived from the JET-ILW L-mode power scans (with NBI) at constant $n_{\mathrm{e}}, B_{\mathrm{T}}$ and $I_{\mathrm{P}}$ in $\mathrm{H}$ and $\mathrm{D}$ [7]. We first note that the atomic mass exponents in the two scalings may be different. In order to relate the isotope mass scaling of the dimensionless thermal energy confinement time $\Omega_{\mathrm{i}} \tau_{\mathrm{E}, \mathrm{th}} \sim\left(\rho^{* \alpha \rho} \beta^{\alpha \beta} \nu^{* \alpha \nu} q^{\alpha q} A^{\alpha \mathrm{A}, \mathrm{D}} \ldots\right)$ to that of the dimensional thermal energy confinement time $\tau_{\mathrm{E}, \mathrm{th}} \sim\left(I_{\mathrm{P}}^{\alpha_{\mathrm{I}}} B_{\mathrm{T}}^{\alpha_{\mathrm{B}}} P^{\alpha_{\mathrm{P}}} n_{e}^{\alpha_{n}} A^{\alpha_{\mathrm{A}, \mathrm{E}}} \ldots\right)$, a transformation between 

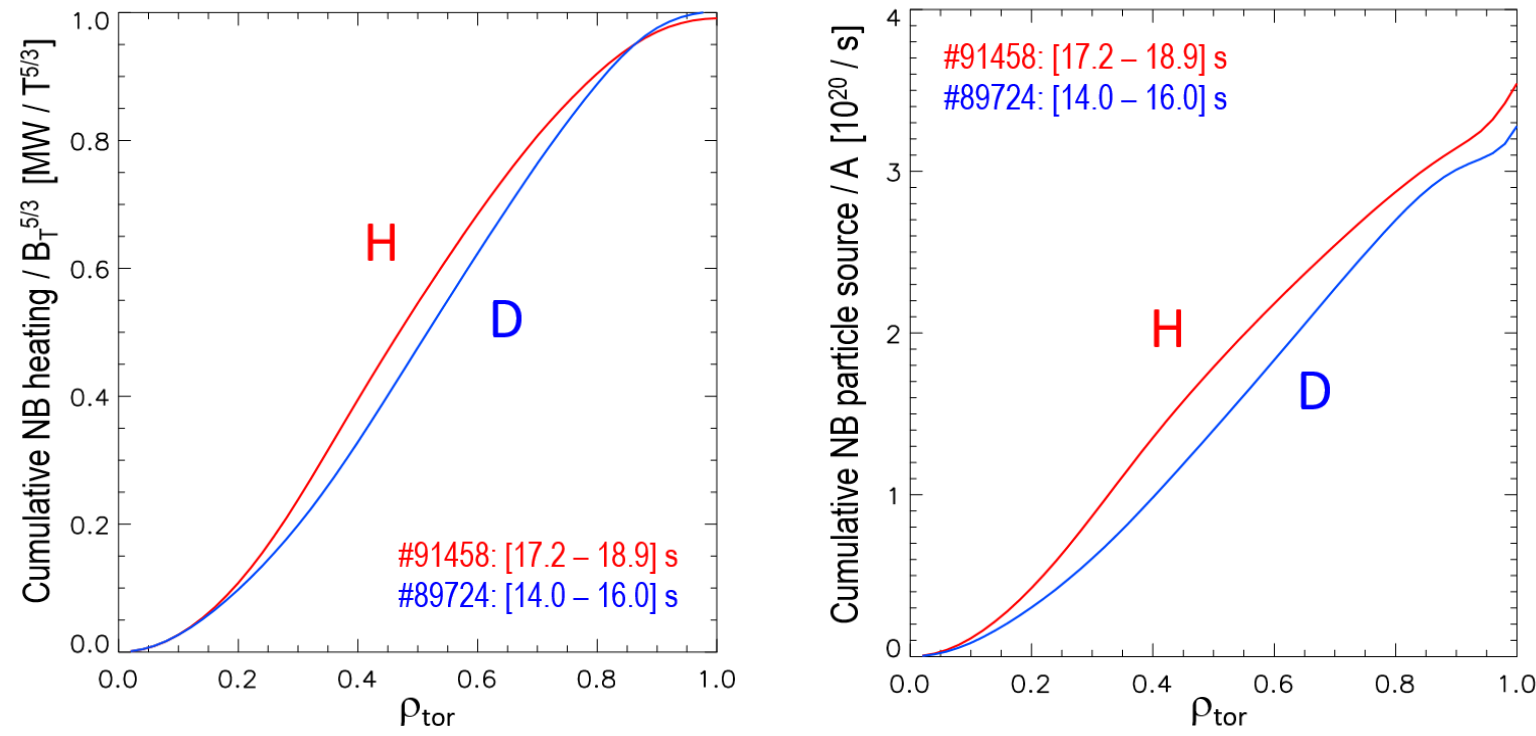

Figure 4. Cumulative volume integral of NB sources for $(a)$ scaled total heating and $(b)$ scaled particle source (TRANSP/NUBEAM) for the $\mathrm{H}$ (red) and D (blue) L-mode isotope identity pair. For each shot, the profiles are time averaged over the steady time window of the discharge, indicated in the plots (see also table 1), corresponding to 100 TRANSP/NUBEAM profiles. 32000 MC markers (at constant census) are used for a single TRANSP/NUBEAM profile.

Table 1. Main parameters of the JET-ILW L-mode isotope identity pair.

\begin{tabular}{lll}
\hline Pulse \# & $\# 91458$ & $\# 89724$ \\
\hline Isotope & $\mathrm{H}$ & $\mathrm{D}$ \\
Time interval (s) & $17.2-18.9$ & $14.0-16.0$ \\
$B_{\mathrm{T}}(\mathrm{T})$ & 1.74 & 2.95 \\
$I_{\mathrm{P}}(\mathrm{MA})$ & 1.44 & 2.46 \\
$P_{\text {abs }}(\mathrm{MW})( \pm 10 \%)$ & 2.56 & 6.24 \\
$\tau_{\mathrm{E}, \mathrm{th}}(\mathrm{s})( \pm 10 \%)$ & 0.155 & 0.19 \\
$\boldsymbol{P}_{\text {abs }} / \boldsymbol{B}_{\mathbf{T}}^{\mathbf{5 / 3}}\left(\mathbf{M W} \mathbf{T}^{-\mathbf{5} / \mathbf{3}}\right)$ & $\mathbf{1 . 0 2}$ & $\mathbf{1 . 0 3}$ \\
$\boldsymbol{Z}_{\text {eff }}( \pm 10 \%)$ & $\mathbf{1 . 4}$ & $\mathbf{1 . 3 5}$ \\
$\boldsymbol{T}_{\mathbf{i}} / \boldsymbol{T}_{\mathbf{e}}$ & $\mathbf{1 . 0}$ & $\mathbf{1 . 0}$ \\
$\boldsymbol{B}_{\mathbf{T}} \tau_{\mathbf{E}, \text { th }} / \mathbf{A}(\mathbf{T s})$ & $\mathbf{0 . 2 7}$ & $\mathbf{0 . 2 8}$ \\
\hline
\end{tabular}

scaling laws in dimensionless and dimensional variables needs to be applied (see table 5 in [2]). The exponent of the isotope mass scaling in dimensional form is [2]:

$\alpha_{\mathrm{A}, \mathrm{E}}=\left[\frac{1}{2} \alpha_{\rho}+\alpha_{\mathrm{A}, \mathrm{D}}+1\right] /\left[1-\frac{1}{2} \alpha_{\rho}-\alpha_{\beta}+2 \alpha\right]$.

Therefore, evaluation of $\alpha_{\mathrm{A}, \mathrm{E}}$ requires, in addition to $\alpha_{\mathrm{A}, \mathrm{D}}$, the knowledge of the scalings of $\Omega_{\mathrm{i}} \tau_{\mathrm{E}, \text { th }}$ with $\rho^{*}, \beta$ and $\nu^{*}$, which were not part of the scope of the experiment presented here. If we tentatively assume from the literature (see e.g. [2] and references therein) that the L-mode scalings are close to Bohm scaling, $\Omega_{\mathrm{i}} \tau_{\mathrm{E}, \mathrm{th}} \sim \rho^{*-2}$, and that both $\beta$ and $\nu^{*}$ scalings are weak in L-mode, namely $\alpha_{\beta} \sim \alpha_{\nu} \sim 0$, then we obtain $\alpha_{\mathrm{A}, \mathrm{E}} \sim 0$. This is not inconsistent with the weak, favourable isotope mass scaling of $\tau_{\mathrm{E} \text {,th }}$ found in the JET-ILW dimensional L-mode scaling in [7]. In those experiments, the anomalous heat and particle diffusivities, $\mathrm{D}_{\perp}$ and $\chi_{\perp}$, were found to be larger in $\mathrm{H}$

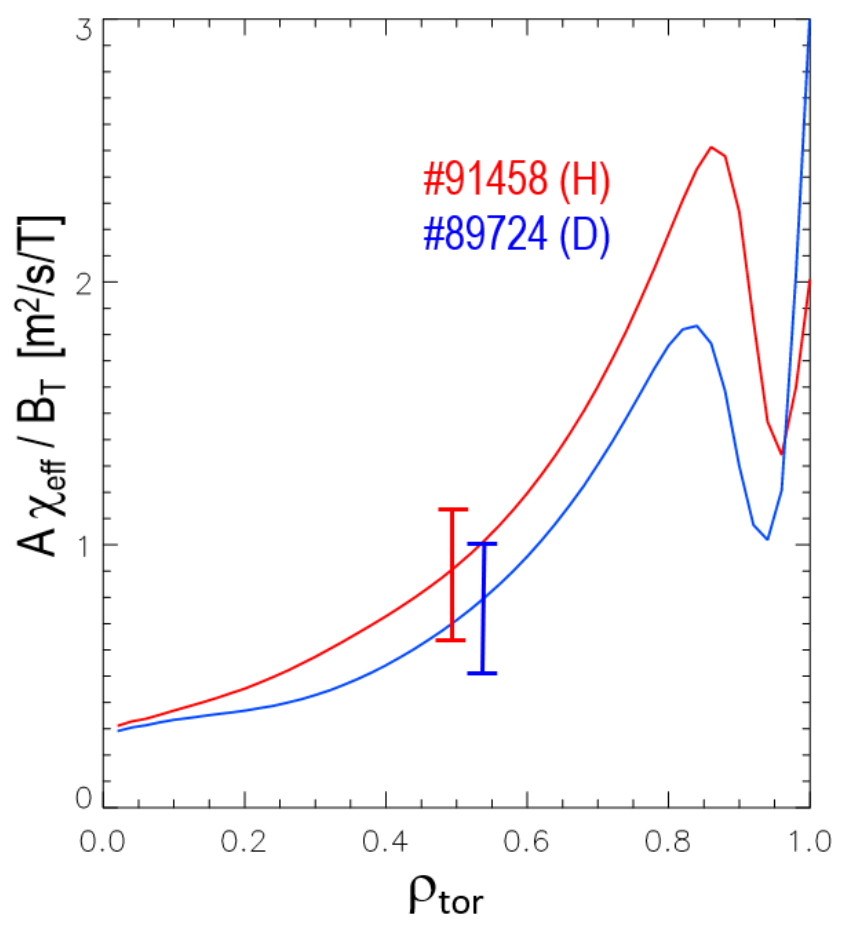

Figure 5. Scaled effective heat diffusivity $A \chi_{\text {eff }} / B_{\mathrm{T}}$ for the L-mode isotope identity pair in $\mathrm{H}$ (red) and $\mathrm{D}$ (blue), with parameters as in table 1 .

than in D only in the edge region (both inside and outside the LCFS) in interpretative EDGE2D/EIRENE simulations [7], while in the plasma core the one-fluid, effective heat diffusivity $\chi_{\text {eff }}\left(\rho_{\text {tor }} \sim 0.5\right)$ was comparable for $\mathrm{H}$ and $\mathrm{D}$ at all power levels of the NBI power scan at constant density [7]. 

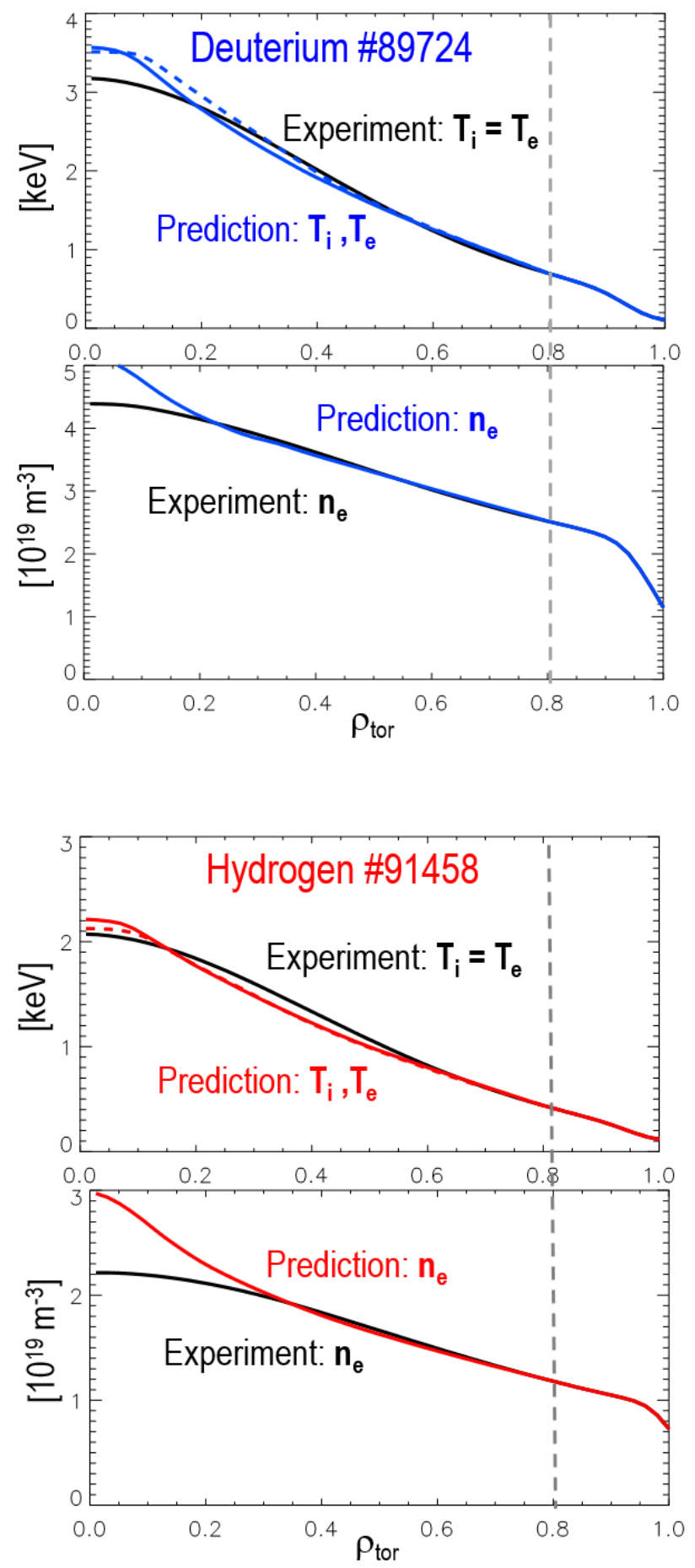

Figure 6. JETTO-TGLF (SAT1) predicted profiles for $T_{\mathrm{e}}, T_{\mathrm{i}}$ and $n_{\mathrm{e}}$ and comparison with experiment (black) for the L-mode isotope identity pair in $\mathrm{H}$ (red) and $\mathrm{D}$ (blue). The model prediction applies to the region between $\rho_{\text {tor }}=0.8$ (vertical, dashed grey line), where the boundary condition is set from experiment, and the sawtooth inversion radius at $\rho_{\text {tor }} \sim 0.2$ (determined from ECE). $T_{\mathrm{i}}=T_{\mathrm{e}}$ from experiment; predicted $T_{\mathrm{e}}=$ solid line, predicted $T_{\mathrm{i}}=$ dashed line in the JETTO-TGLF simulations. No sawtooth model was used here.

\section{Predictive core plasma transport modelling}

Predictive core plasma transport modelling of the L-mode isotope identity pair in $\mathrm{H}$ and $\mathrm{D}$ was carried out with flux-driven JETTO-TGLF using the multi-scale saturation rule including
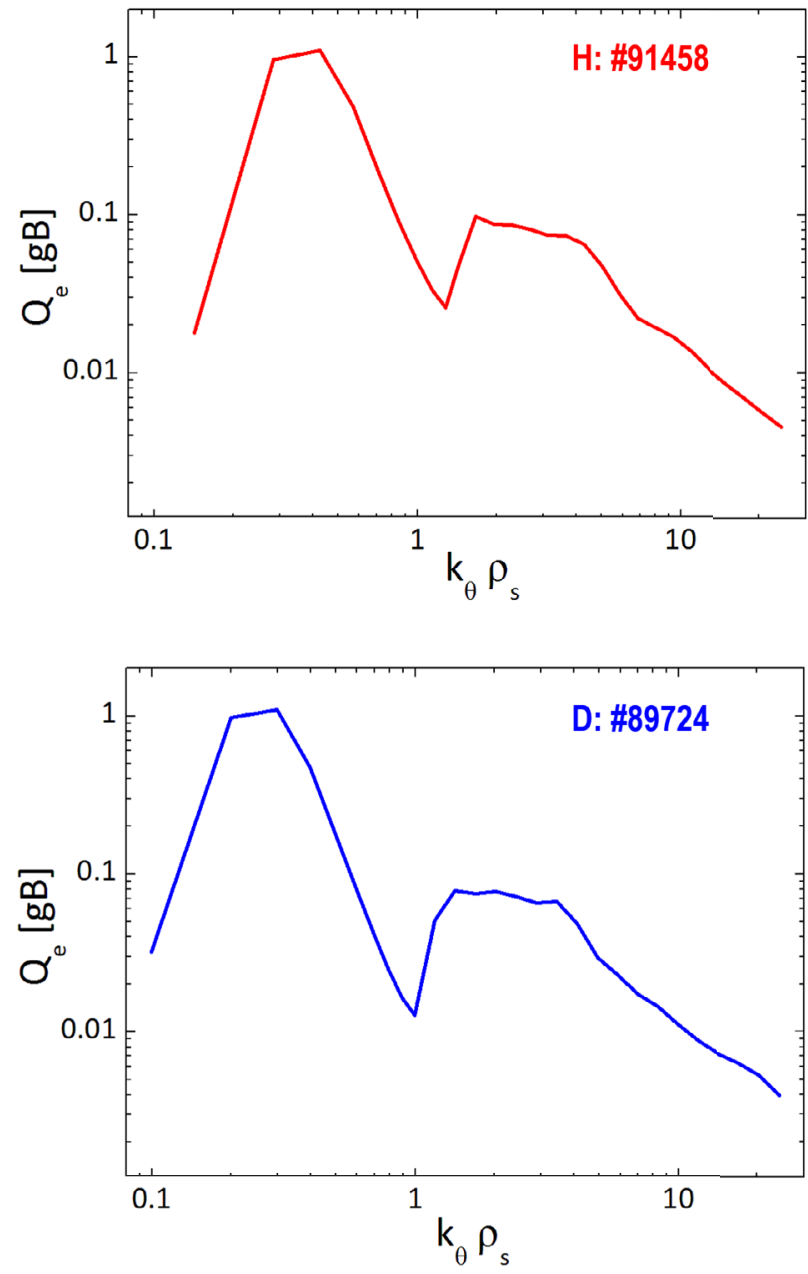

Figure 7. Electron heat flux spectra (in gyro-Bohm units, normalized to D mass) from TGLF at $\rho_{\text {tor }}=0.56$, for H (\#91458) and D (\#89724) L-mode shots, corroborating the finding that ITGs are the dominant instabilities in the plasma core, for both isotopes.

ion and electron transport scales (SAT1) [15]. The model predictions apply to the plasma region between $\rho_{\text {tor }}=0.8$, where the boundary conditions are set from experiment, and the sawtooth inversion radius $\rho_{\text {tor }} \sim 0.2$. No sawtooth model was used in this work. Inputs to the local, quasilinear turbulence model TGLF [16] are the local values of $T_{\mathrm{i}}, n_{\mathrm{i}}, T_{\mathrm{e}}, n_{\mathrm{e}}, v_{\text {tor }}$ (toroidal rotation) and the local gradients, as well as local $q$, magnetic shear, plasma elongation and triangularity. The fluxes, generated as part of the output, are used as input to the transport code JETTO [17, 18], which solves the 1D transport equations (averaged over the magnetic surfaces) for electron and ion densities and energies in a time dependent axisymmetric MHD equilibrium configuration. Additional inputs to JETTO are the boundary conditions (from experiment in this case) and the heat and particle sources/sinks. In the simulations reported here, the NBI heat and particle sources are computed by TRANSP/NUBEAM [13]. The main output is the prediction of $T_{\mathrm{i}}, T_{\mathrm{e}}, n_{\mathrm{i}}, n_{\mathrm{e}}$ profiles, while $v_{\text {tor }}$ is not predicted in this case and is an input from experiment. While a single call to TGLF is a gradient-driven turbulence simulation, the coupled JETTO-TGLF runs evolve the profiles from the initial condition provided by experiment using the fluxes computed by 

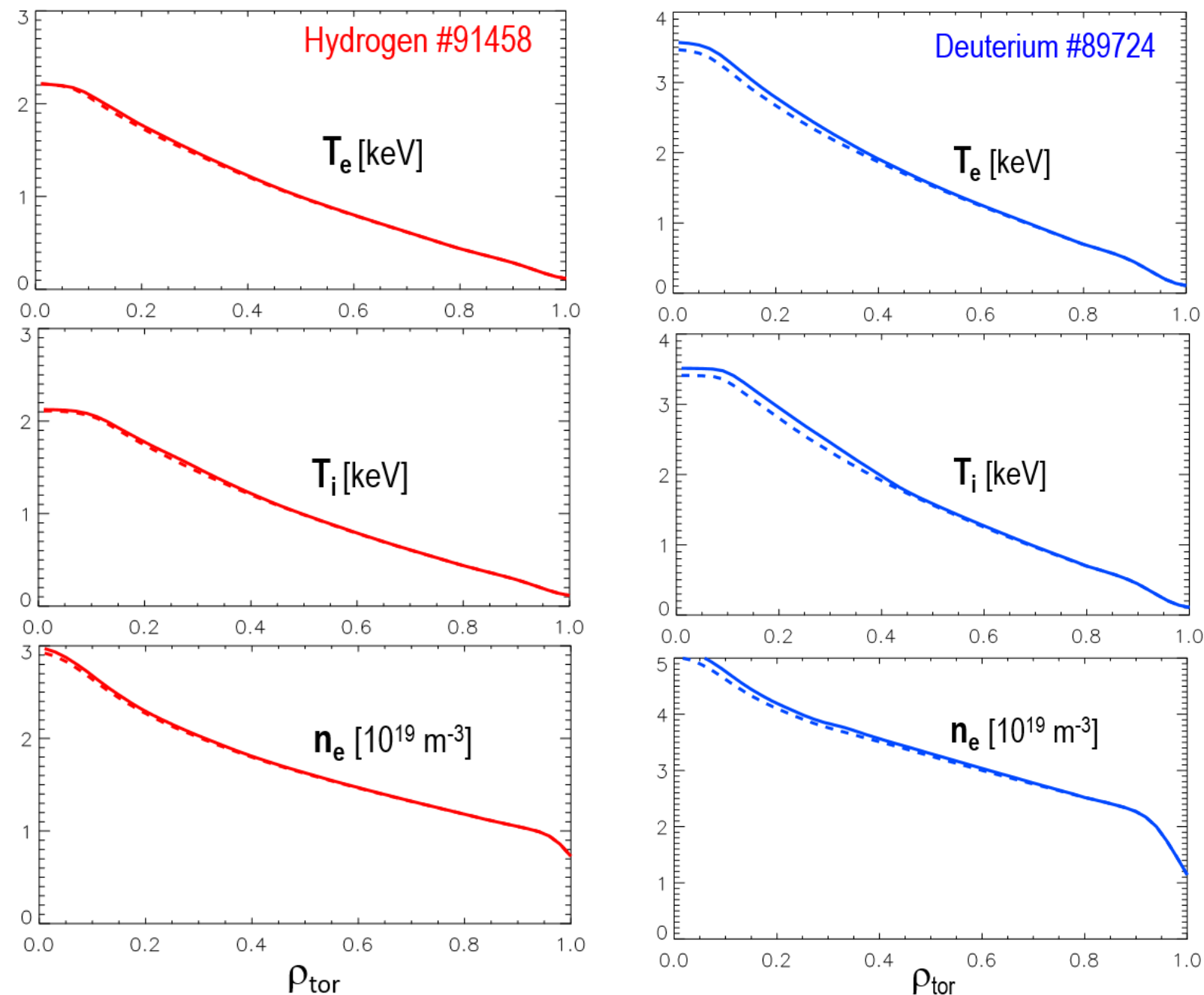

Figure 8. JETTO-TGLF (SAT1) predicted profiles for $T_{\mathrm{e}}, T_{\mathrm{i}}$ and $n_{\mathrm{e}}$ with (solid lines) and without (dashed lines) $E \times B$ shear effectsresulting from the experimentally measured $v_{\text {tor }}$ gradients-for the $\mathrm{H}$ (red) and $\mathrm{D}$ (blue) L-mode isotope identity pair.

TGLF. In this framework, TGLF is called repeatedly until a steady state is reached (after a simulation time $\gg \tau_{\mathrm{E}, \mathrm{th}}$ ). The final profiles predicted by JETTO thus represent a self-consistent flux-driven prediction of TGLF, with the fluxes in balance with the sources and sinks.

The model prediction of the L-mode isotope identity pair is in very good agreement with experiment for both isotopes, as shown in figure 6. With fixed gradient inputs, the local TGLF model has gyro-Bohm scaling of the fluxes. However, when it is used (within JETTO) to make flux driven global predictions, the stiff core heat transport, intended here in terms of strong sensitivity of the applied heat fluxes to small variations in the core temperature gradients, which is typical of JET-ILW NBI heated L-modes and $\mathrm{H}$-modes at moderate input powers $\left(P_{\mathrm{NBI}}<20 \mathrm{MW}\right)$, see e.g. [7], overcomes the local gyro-Bohm scaling $\left(\chi_{\mathrm{gB}} \sim \sqrt{ } A\right)$ by the same argument laid out in [19] in the prediction of the kinetic profiles and thus of the global thermal energy confinement time (once the boundary conditions at the edge are imposed from experiment). In other words, the local gyro-Bohm scaling of core heat transport does not simplistically translate to the global energy confinement time as $\tau_{\mathrm{E}, \mathrm{th}} \sim$ $a^{2} / \chi_{\mathrm{gB}} \sim 1 / \sqrt{ } A$ (against much experimental evidence), since the heat diffusivity is not constant but strongly depends on the temperature gradient.

In the TGLF simulations reported here, ion temperature gradient modes (ITGs) are found to be the dominant instabilities in the plasma core of both $\mathrm{H}$ and D L-modes, both at ion spatial scales $\left(0<k_{\theta} \rho_{\mathrm{s}}<1\right)$-as shown by the growth rates and frequencies of the two most dominant modes-and at electron spatial scales $\left(k_{\theta} \rho_{\mathrm{s}} \gg 1\right)$, where most of the electron heat flux is found at ion scales, as indicated by the electron heat flux spectra of figure 7 . Furthermore, the ratio of $\left(\gamma_{\mathrm{ETG}} / k_{\mathrm{ETG}}\right) /$ $\left(\gamma_{\mathrm{ITG}} / k_{\mathrm{ITG}}\right)$ is 0.62 for \#89724 and 0.55 for \#91458. Further confirmation that ITGs are the dominant instabilities in the plasma core of these isotope identity L-modes is obtained by the JETTO-TGLF power balance, which yields a ratio of ion to electron heat flux at mid-radius $Q_{\mathrm{i}} / Q_{\mathrm{e}}\left(\rho_{\mathrm{tor}}=0.5\right)=2$ for the $\mathrm{H}$ shot $(\# 91458)$ and $Q_{\mathrm{i}} / Q_{\mathrm{e}}\left(\rho_{\text {tor }}=0.5\right)=2.5$ for the $\mathrm{D}$ shot (\#89724).

Effects of collisions, toroidal rotation and and $E \times B$ shearing are included in the quasi-linear computations, while effects of impurities are not included. We note, however, that $Z_{\text {eff }} \sim 1.4$ for these plasmas, with Be the main impurity, for both $\mathrm{H}$ and D. Stabilization of ITG modes due to core fast 

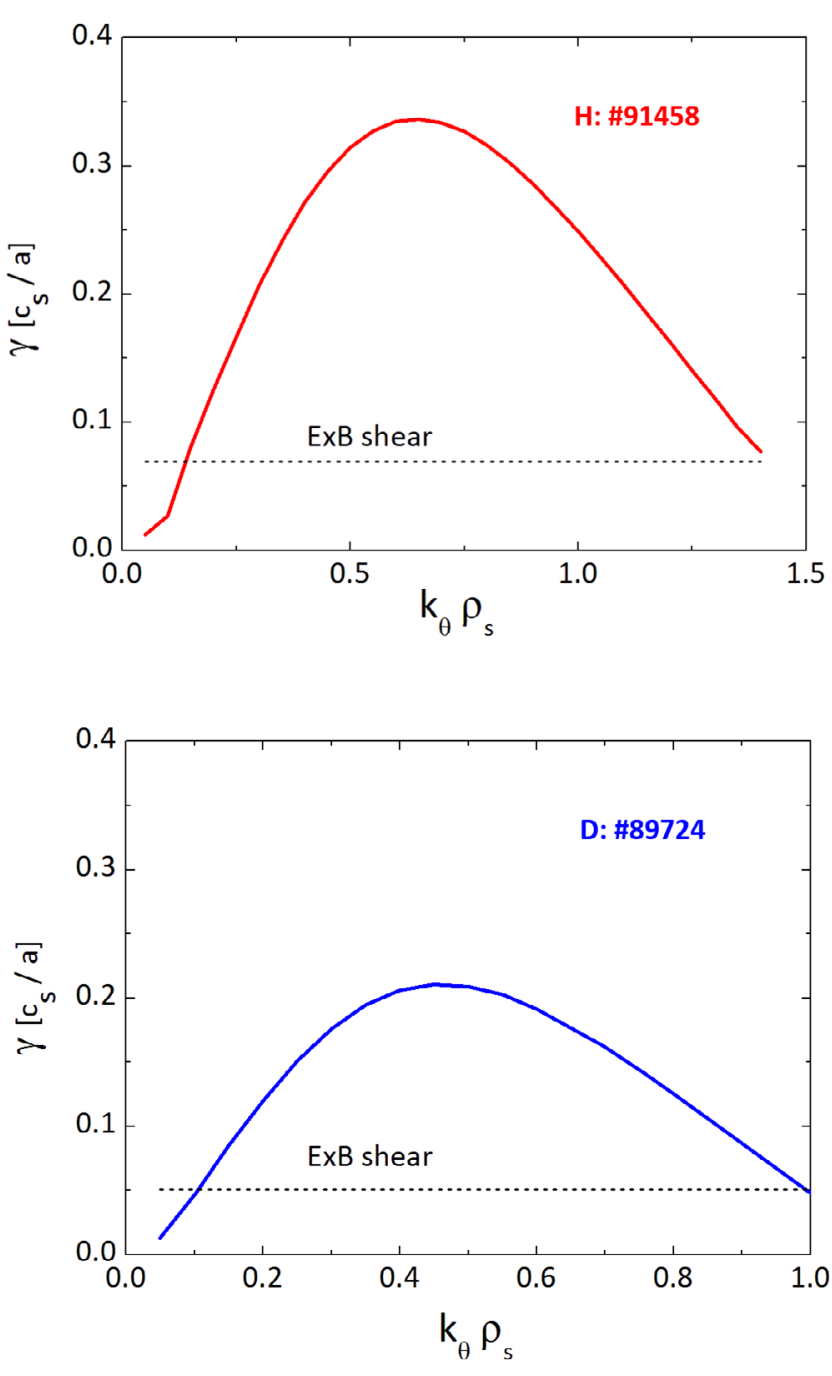

Figure 9. Growth rate of the most dominant mode at ion scales from TGLF predictive runs, for the $\mathrm{H}$ (red) and $\mathrm{D}$ (blue) pulses of the isotope identity pair, as well as the levels of $E \times B$ shear (dashed lines), calculated from the experimentally measured $v_{\text {tor }}$ profiles (rates are in $c_{\mathrm{s}} / a$ units, normalized to Deuterium).

ions pressure gradient is not included here, due to the low fast ion population fraction of these L-mode discharges at low NB heating levels. The JETTO-TGLF runs indicate a negligible effect of toroidal rotation $v_{\text {tor }}$ (which is an input from experiment in this case, with $v_{\text {tor }}$ slightly larger in D than in $\mathrm{H}$ ) and $E \times B$ shearing on the predicted heat and particle transport channels of the isotope identity $\mathrm{H}$ and $\mathrm{D}$ pair, as shown in figure 8 . This is perhaps not surprising, given the low beta $\left(\beta_{\mathrm{N}}\right.$ $\sim 0.6$ ) and low momentum input of these L-mode plasmas. Indeed, both for the $\mathrm{H}$ and $\mathrm{D}$ pulses, the level of $E \times B$ shear is low compared to the growth rate of the most dominant mode at ion scales obtained from the TGLF predictive runs, as shown in figure 9. Therefore, the mismatch in Mach number between the $\mathrm{H}$ and $\mathrm{D}$ shots, quoted in section 2, is not significant in this identity pair and does not invalidate the achieved dimensionless profiles identity.

The $\mathrm{H}$ and $\mathrm{D}$ identity pair have, by definition, very similar core density profile peaking (see figure 1(a)) at same $\rho^{*}$, $\nu^{*}, \beta$ and $q$. As noted in section 2, the scaled NBI particle source profile is, however, somewhat larger in $\mathrm{H}$ than in $\mathrm{D}$ for $\rho_{\text {tor }}<0.5$. The JETTO-TGLF predictive modelling indicates that, in addition to the pinch term, the NBI particle source also contributes to the core density peaking of these L-modes, by approximately $40 \%$, after subtraction of the edge contribution. Recent theoretical studies of the dependence of turbulent particle flux on isotope mass induced by collisionality for ITG dominated transport lead to the expectation that the peaking of density profiles of $\mathrm{H}$ plasmas be slightly higher than those of D plasmas-but inside typical density profile measurements error bars - at similar plasma parameters and without a significant particle source [20]. This result is also found in the JETTO-TGLF predictive modelling reported here. This is illustrated in figures $10(a)$ and $(b)$, where the predicted kinetic profiles (dashed lines) are compared with experiment (solid lines) in JETTO-TGLF simulations where the isotope mass is swapped in the transport calculations (D into H for \#89724 and H into D for \#91458), while boundary conditions and sources and sinks are kept from experiment. It can be seen that the predicted $n_{\mathrm{e}}$ profile for $\mathrm{D}$ with $A=1$ (figure $10(a)$ ) is slightly more peaked than in figure $6(a)$, while no appreciable difference is observed in the predicted temperature profiles.

\section{Conclusions and outlook}

Isotope identity experiments test the validity of the confinement scale invariance principle, which is at the heart of predictive capability for fusion performance in future devices. In particular, with such experiments one can test whether the same physics is involved when varying $\rho^{*}$ via $\left\{B_{\mathrm{T}}, T\right\}$ only, as in $\rho^{*}$-scaling experiments (see e.g. the review work of [2] and references therein) which are typically run in D plasmas, as opposed to via $\left\{B_{\mathrm{T}}, T\right.$ and $\left.A\right\}$ as in the experiments of [3] in JET-C and those reported here for JET-ILW.

The studies reported in this paper have shown that NBIheated L-mode plasmas have been obtained in JET-ILW in $\mathrm{H}$ and $\mathrm{D}$, with matched profiles of the dimensionless plasma parameters, $\rho^{*}, \nu^{*}, \beta$ and $\mathrm{q}$ in the plasma core confinement region and same scaled energy confinement time $\Omega_{\mathrm{i}} \tau_{\mathrm{E} \text {,th }}$. Therefore, in this region of the plasma, where ITGs are the dominant instability, the confinement scale invariance principle is satisfied. The dimensionless thermal energy confinement time, $\Omega_{\mathrm{i}} \tau_{\mathrm{E}, \mathrm{th}}$, and the scaled core plasma heat diffusivity $A \chi_{\mathrm{eff}} / B_{\mathrm{T}}$, are matched in $\mathrm{H}$ and $\mathrm{D}$, yielding $\Omega_{\mathrm{i}} \tau_{\mathrm{E}, \mathrm{th}} \sim A^{0.05 \pm 0.1}$. Predictive modelling with JETTO-TGLF of the isotope identity pair is in very good agreement with experiment for both isotopes, for both particle and energy channels. The stiff core heat transport, which is typical of JET-ILW NBI heated L-modes, such as those of this study, overcomes the local gyro-Bohm scaling of gradient-driven TGLF, explaining the absence of isotope mass dependence of the dimensionless thermal energy confinement time $\Omega_{\mathrm{i}} \tau_{\mathrm{E}, \mathrm{th}}$. The effect of $E \times B$ shearing on the predicted heat and particle transport channels is found to be negligible for these low beta and low momentum input plasmas (while this effect may instead be sizeable in high power H-modes, see e.g. [21]), hence the mismatch in 

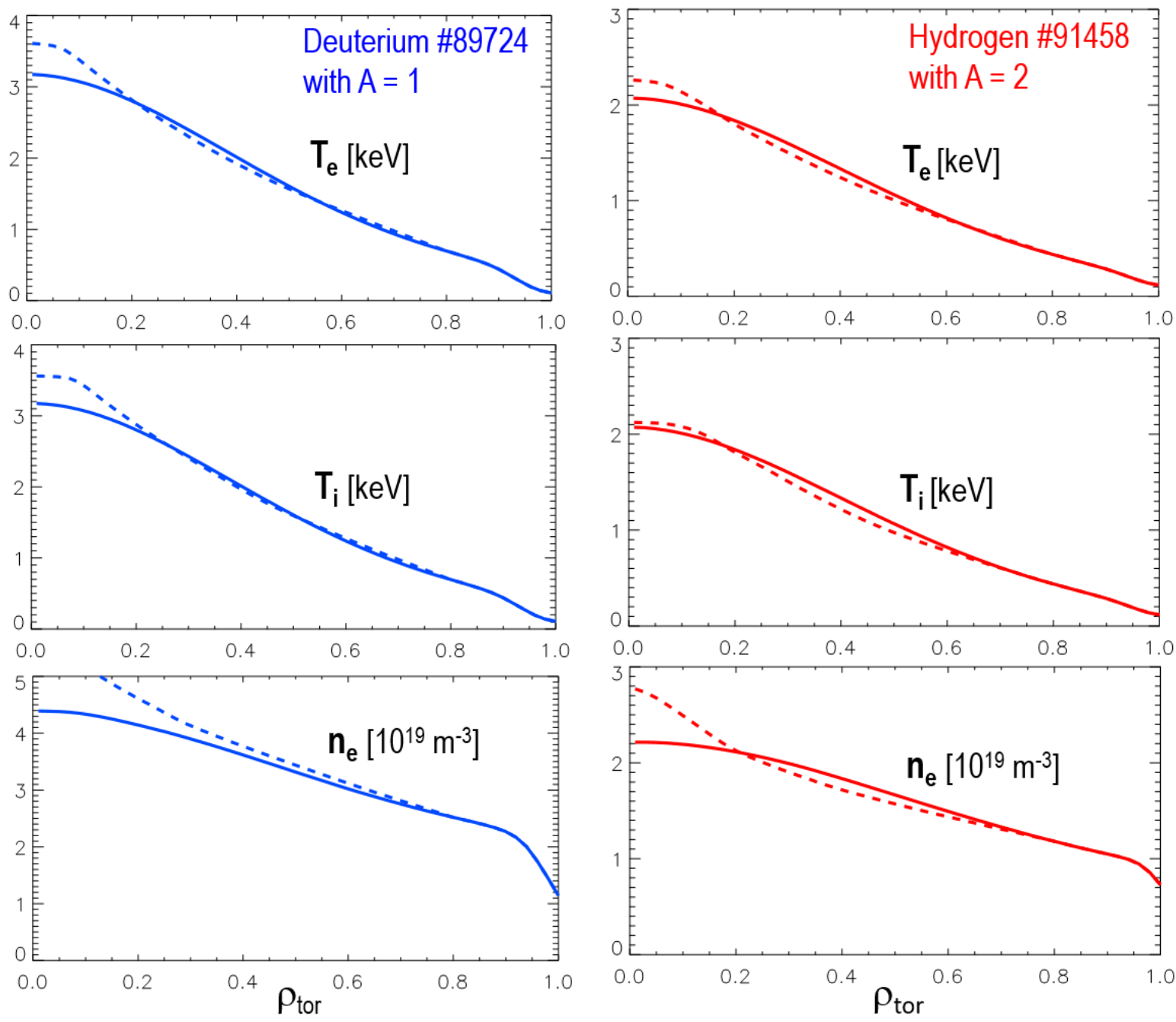

Figure 10. $T_{\mathrm{e}}, T_{\mathrm{i}}$ and $n_{\mathrm{e}}$ experimental profiles (solid lines) and JETTO-TGLF (SAT1) predicted profiles (dashed lines) where the isotope mass is swapped in the transport calculations (left: $\mathrm{D} \rightarrow \mathrm{H}$ and right: $\mathrm{H} \rightarrow \mathrm{D}$ ), while boundary conditions and sources/sinks are input from experiment for $\mathrm{H}$ shot \#91458 (red) and D shot \#89724 (blue); to be compared with the profiles of figure 6.

the $M$ number profile does not invalidate the isotope identity in this case.

An isotope identity in $\mathrm{H}$ and $\mathrm{D}$ had been achieved in the type I ELMy H-mode regime in JET with C-wall, remarkably throughout the entire plasma radius. In the L-mode isotope identity reported in this paper, density and temperature measurements with HRTS are not available inside the sawtooth inversion radius $\left(\rho_{\text {tor }} \sim 0.2\right)$. However, the scaled density profiles from LIDAR [22] and temperature profiles from ECE [23], which extend radially inwards to $\rho_{\text {tor }}=0$, are very similar in $\mathrm{H}$ and $\mathrm{D}$, indicating that the isotope identity may also have been achieved in the plasma centre. In this region, though, the scaled sawtooth frequency is not matched, being almost twice in $\mathrm{H}$ than in D, unlike in the JET-C isotope identity in $\mathrm{H}$-mode. As the volume of the sawtooth dominated region is small compared to that of the confinement region, it has little impact on the global energy confinement time, which, together with core plasma transport, is the focus of this work. We're also not venturing here to explore whether the $\mathrm{H}$ and $\mathrm{D}$ scaled edge kinetic profiles are matched near the last closed flux surface (LCFS) and in the SOL, as this goes beyond the accuracy of the available measurements for this $\mathrm{H}$ \& D pair in this plasma region, where HRTS signal statistics becomes poor and the ECE diagnostic spatial resolution is inadequate to resolve the temperature gradient (and, in addition, transition to optically thin plasma makes the $T_{\mathrm{e}}$ profile from ECE unreliable in this region). On the other hand, the narrow edge layer just inside and outside the LCFS is the crucial one where isotope dependencies may occur in L-mode transport, in particular for the particle channel in JET-ILW, as discussed in [7]. This is the subject of further, ongoing study and has important implications for the understanding of the isotope dependence of the L-H power threshold and of $\mathrm{H}$-mode pedestal transport.

Last, but not least, planned JET-ILW experiments in $T$ are crucial, not only to add a 3rd isotope to the dataset (by far a non-trivial experimental achievement per se), but because the physics underlying the 'isotope effect' is complex and non-linear: it involves interaction between the local scales, where gradient driven instabilities arise, and global scales, 
where stiff heat transport and density profile peaking are important. As $T$ plasmas are expected to have similar or better confinement than D, and not worse as in H [7], studying D and $\mathrm{T}$ means focussing on the fusion-relevant isotope pair. We also stress again that the physics mechanisms that play a role in explaining the isotope mass dependence of L-mode core transport, such as those discussed in this paper, are not necessarily the same as those responsible for explaining core transport in high power H-modes. An obvious example is the effect of shear rate, which is predicted to reduce transport as the isotope mass is increased: the instabilities growth rate is reduced as $\gamma_{\max } \sim 1 / \sqrt{ } A$, for fixed profile shapes [19, 21, 24]. Therefore, the stabilizing effect of $E \times B$ shear should be assessed realistically in conjunction with the profile shape effects as the isotope mass is changed in experiment.

\section{Acknowledgments}

This work was carried out within the framework of the EUROfusion Consortium and received funding from the Euratom research and training programme 2014-2018 under grant agreement No. 633053 and from the RCUK Energy Programme grant No. EP/P012450/1. The views and opinions expressed herein do not necessarily reflect those of the European Commission. The authors are grateful to Drs Clive Challis, Yann Camenen and Martin Valovic for very useful discussions.

\section{ORCID iDs}

C.F. Maggi (10 https://orcid.org/0000-0001-7208-2613

F. Auriemma (i) https://orcid.org/0000-0002-1043-1563

F. Eriksson (1) https://orcid.org/0000-0002-2740-7738

L. Horvath $\mathbb{0}$ https://orcid.org/0000-0002-5692-6772

\section{References}

[1] Connor J.W. and Taylor J.B. 1977 Nucl. Fusion 171047

[2] Luce T.C. et al 2008 Plasma Phys. Control. Fusion 50043001

[3] Cordey J.G. et al 2000 Plasma Phys. Control. Fusion 42 A127

[4] Brezinsek S. et al 2013 Nucl. Fusion 53083023

[5] Cordey J.G. et al 1999 Nucl. Fusion 39301

[6] Pasqualotto R. et al 2004 Rev. Sci. Instrum. 753891

[7] Maggi C.F. et al 2018 Plasma Phys. Control. Fusion 60014045

[8] Delabie E. et al 2014 Overview and interpretation of L-H threshold experiments on JET with the ITER-like wall 25th IAEA Int. Conf. on Fusion Energy (St Petersburg, 2014) EX/P5-24 (www-pub.iaea.org/iaeameetings/46091/25thFusion-Energy-Conference-FEC-2014)

[9] Nunes I. et al 2016 First results from recent JET experiments in hydrogen and hydrogen-deuterium plasmas Preprint: 2016 IAEA Fusion Energy Conference (Kyoto, Japan, 17-22 October 2016) EXC-P8 (www-pub.iaea.org/ iaeameetings/48315/26th-IAEA-Fusion-Energy-Conference)

[10] Menmuir S. et al 2014 Rev. Sci. Instrum. 85 11E412

[11] Ho A. et al 2018 Turbulent transport model validation at JET using integrated modelling enhanced by Gaussian process regression 45th EPS Conf. on Plasma Physics (Prague, Czech Republic, 2-6 July 2018) (http://ocs.ciemat.es/ EPS2018PAP/pdf/P4.1088.pdf)

[12] Challis C.C. et al 1989 Nucl. Fusion 29563

[13] Goldston R.J. et al 1981 J. Comput. Phys. 4361

[14] Tibone F. et al 1993 Nucl. Fusion 331319

[15] Staebler G. et al 2017 Nucl. Fusion 5766046

[16] Staebler G. et al 2007 Phys. Plasmas 14055909

[17] Cenacchi G. and Taroni A. 1988 JET internal report 03

[18] Romanelli M. et al 2014 Plasma Fusion Res. 93403023

[19] Bateman G. et al 1999 Phys. Plasmas 64607

[20] Angioni C. et al 2018 Phys. Plasmas 25082517

[21] Garcia J. et al 2017 Nucl. Fusion 57014007

[22] Maslov M. et al 2013 JINST 8 C11009

[23] De la Luna E. et al 2004 Rev. Sci. Instrum. 753831

[24] Scott S. et al 1996 Proc. 16th Fusion Energy Conf. vol 1 (Montreal, Canada, 7-11 October 1996) p 573

[25] Litaudon X. et al 2017 Nucl. Fusion 57102001 